\title{
Catalytic Mechanism and Product Specificities of Protein Arginine Methyltransferase PRMT7: A Study from QM/MM molecular dynamics and free energy simulations
}

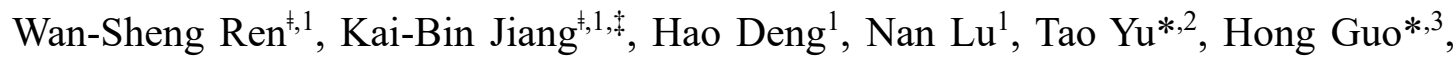
Ping Qian*,1

${ }^{1}$ Chemistry and Material Science Faculty, Shandong Agricultural University, Taian 271018, P. R. China

${ }^{2}$ Department of Chemistry, University of North Dakota, Grand Forks, ND 58202-9024, United States

${ }^{3}$ Department of Biochemistry and Cellular and Molecular Biology, University of Tennessee, Knoxville, TN 37996, United States UT/ORNL Center for Molecular Biophysics, Oak Ridge National Laboratory, Oak Ridge, TN 37830, United States

${ }^{\ddagger}$ These authors contributed equally to this work.

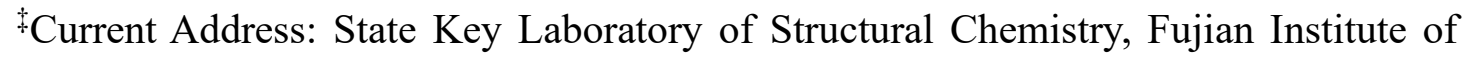
Research on the Structure of Matter, Chinese Academy of Sciences, 350002, P. R. China.

${ }^{*}$ Corresponding authors: Tel.: +86 538 8241175; fax: +86 5388242251.

E-mail address: qianp@sdau.edu.cn (to P. Qian), hguo1@utk.edu (to H. Guo) and tao.yu.1@und.edu (to T. Yu) 
Supporting Information 
Comparison of potential energy curves for the methyl transfer calculated from DFTB3, MP2/6-31G(d) and MP2/6-31G(d,p) in simplified cluster models

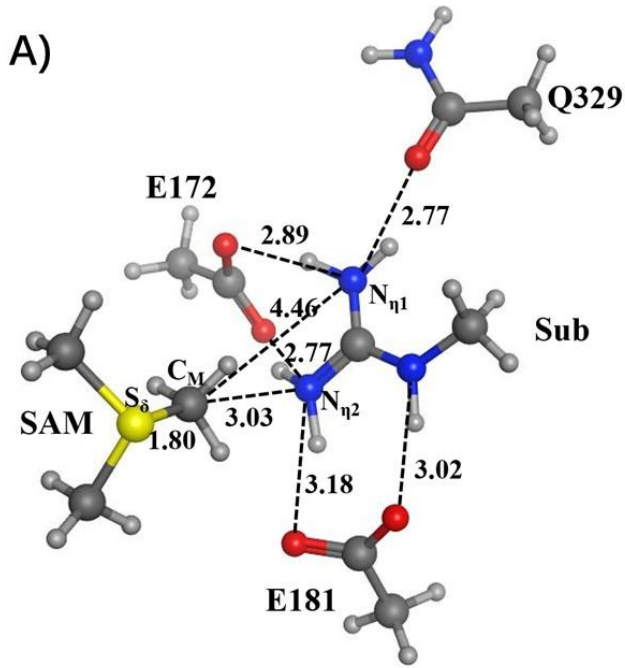

RS

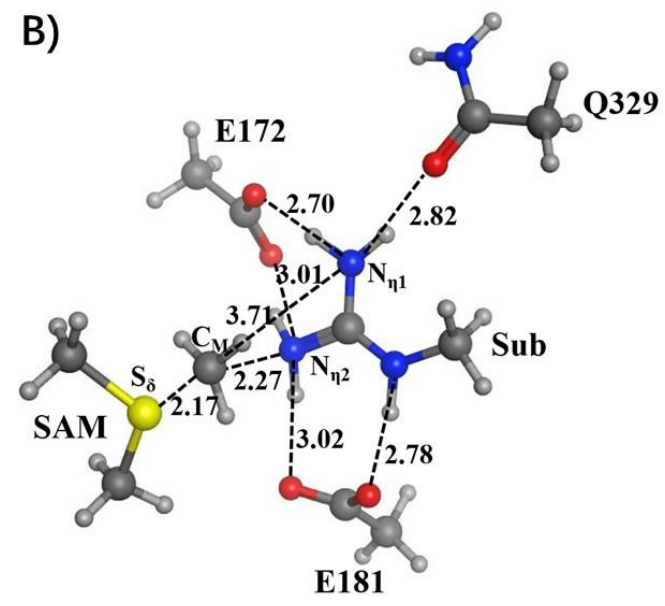

TS

C)

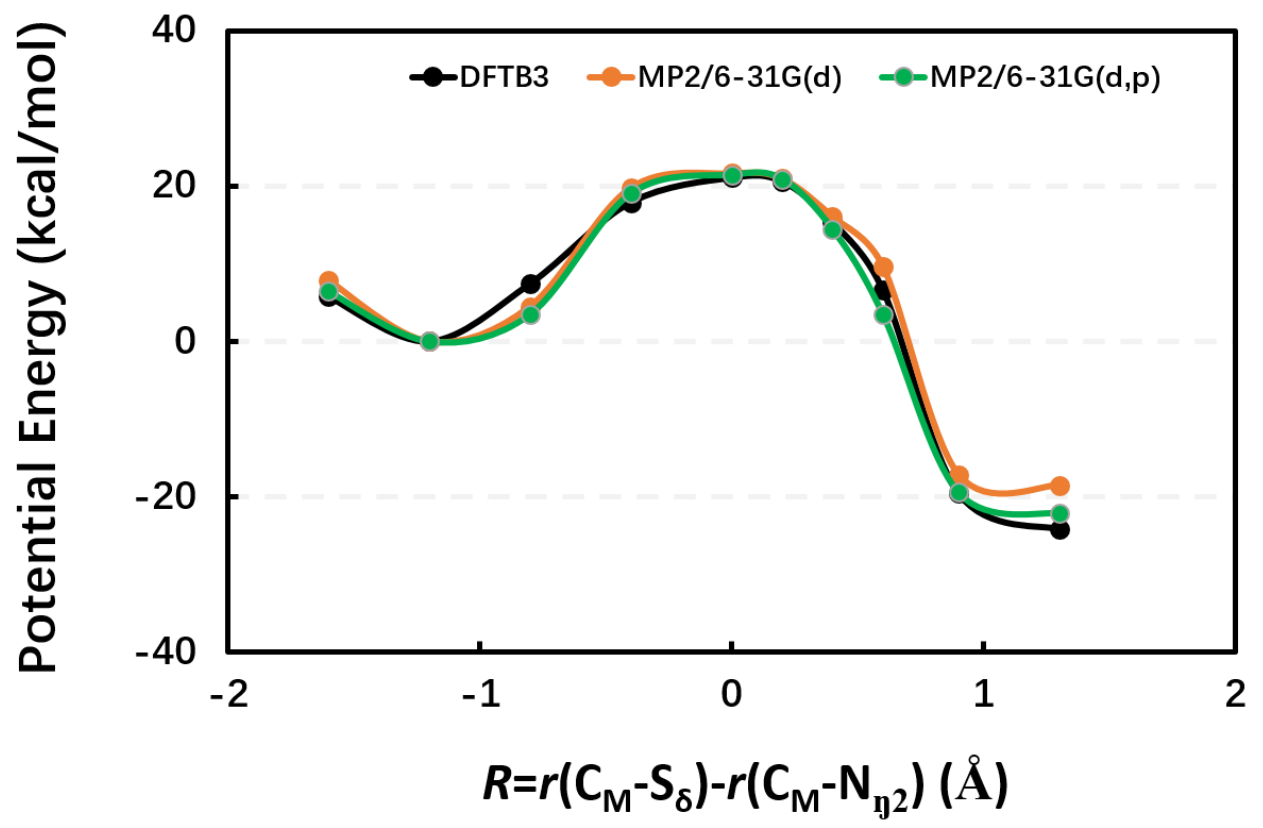

Figure S1. (A) The cluster model system for the reaction state (RS) of wild-type PRMT7 used for comparison the results of potential energy calculations from DFTB3, MP2/6-31G(d) and MP2/6-31(d,p) calculations. The methyl group was transferred from the $\mathrm{S}_{\delta}$-atom to the guanidine $\mathrm{N}_{\eta 2}$-atom. (B) The structure near transition state (TS) for the methyl transfer. (C) Comparison of potential energy curves for the methyl transfer calculated from DFTB3, MP2/6-31G(d) and MP2/6-31G(d,p), respectively. 
Comparison of the Mulliken charge distributions from the DFTB3/CHARMM calculations for guanidino group in wild-type PRMT7 and three mutants (E172Q, E181Q, Q329A) as well as Mulliken charge distributions from the HF/STO-3G calculations for guanidino group in four corresponding models

Table S1. Comparison of the Mulliken charge distributions (unit: e) of guanidino group in wild-type PRMT7 and three mutants based on the DFTB3/CHARMM calculations using typical structures of the wild-type and mutated enzymes obtained from the QM/MM MD simulations.

\begin{tabular}{|c|c|c|c|c|c|}
\hline $\begin{array}{c}\text { Molecular } \\
\text { Label }^{\mathrm{a}}\end{array}$ & $\begin{array}{c}\text { Atomic } \\
\text { Label }^{\mathrm{a}}\end{array}$ & WT & E172Q & E181Q & Q329A \\
\hline \multirow{9}{*}{ Sub } & NE & -0.31290 & -0.28172 & -0.22043 & -0.2659 \\
\hline & HE & 0.30546 & 0.28319 & 0.24919 & 0.28652 \\
\hline & $\mathrm{CZ}$ & 0.52443 & 0.49332 & 0.47848 & 0.52305 \\
\hline & $\mathbf{N}_{n} 1$ & -0.43220 & -0.40209 & -0.44412 & -0.44635 \\
\hline & HH11 & 0.25114 & 0.28364 & 0.34175 & 0.32138 \\
\hline & HH12 & 0.30188 & 0.27575 & 0.30072 & 0.23625 \\
\hline & $\mathbf{N}_{n 2}$ & -0.51577 & -0.38762 & -0.45007 & -0.50241 \\
\hline & HH21 & 0.27439 & 0.27219 & 0.29295 & 0.31202 \\
\hline & HH22 & 0.32841 & 0.30243 & 0.25216 & 0.28058 \\
\hline \multicolumn{2}{|c|}{ Guanidino group } & 0.72484 & 0.83909 & 0.80063 & 0.74514 \\
\hline
\end{tabular}


Table S2. Comparison of the Mulliken charge distributions (unit: e) in four small model systems (see the structures in Figure S2) based on the HF/STO-3G calculations.

\begin{tabular}{|c|c|c|c|c|c|c|c|c|c|c|c|}
\hline $\begin{array}{c}\text { Molecular } \\
\text { Label }^{\mathrm{a}}\end{array}$ & $\begin{array}{l}\text { Atomic } \\
\text { Label }^{\mathrm{a}}\end{array}$ & WT & E172Q & E181Q & Q329A & $\begin{array}{c}\text { Molecular } \\
\text { Label }^{\mathrm{a}}\end{array}$ & $\begin{array}{c}\text { Atomic } \\
\text { Label }^{\mathrm{a}}\end{array}$ & WT & E172Q & E181Q & Q329A \\
\hline \multirow{13}{*}{ SAM } & $\mathbf{S}_{\delta}$ & 0.493803 & 0.502782 & 0.531077 & 0.491074 & \multirow{13}{*}{ Sub } & $\mathbf{N}_{n 1}$ & -0.44103 & -0.415399 & -0.428612 & -0.441866 \\
\hline & $\mathbf{C}_{M}$ & -0.223326 & -0.214754 & -0.248886 & -0.217716 & & HH11 & 0.290791 & 0.247571 & 0.30255 & 0.280349 \\
\hline & HM1 & 0.117354 & 0.139674 & 0.140417 & 0.139219 & & HH12 & 0.220625 & 0.255683 & 0.289871 & 0.223503 \\
\hline & HM2 & 0.165463 & 0.132322 & 0.140074 & 0.123448 & & $\mathbf{N}_{\mathrm{n} 2}$ & -0.451915 & -0.425013 & -0.430493 & -0.452757 \\
\hline & HM3 & 0.140551 & 0.110346 & 0.134358 & 0.149334 & & HH21 & 0.26869 & 0.236899 & 0.309891 & 0.277465 \\
\hline & CG & -0.22792 & -0.22026 & -0.240321 & -0.239485 & & HH22 & 0.25819 & 0.305989 & 0.221683 & 0.257363 \\
\hline & HG1 & 0.12633 & 0.145203 & 0.128426 & 0.12731 & & $\mathbf{C Z}$ & 0.431032 & 0.451305 & 0.456944 & 0.436781 \\
\hline & HG2 & 0.123034 & 0.113907 & 0.126665 & 0.117441 & & NE & -0.365891 & -0.349418 & -0.345345 & -0.363625 \\
\hline & HG3 & 0.11446 & 0.132871 & 0.14221 & 0.122798 & & HE & 0.282187 & 0.291359 & 0.235998 & 0.29329 \\
\hline & $\mathrm{C5}$ & -0.230397 & -0.2352 & -0.254022 & -0.215606 & & CD & -0.067659 & -0.064206 & -0.06241 & -0.06724 \\
\hline & H5'1 & 0.120141 & 0.138554 & 0.124464 & 0.113092 & & HD1 & 0.078034 & 0.065596 & 0.085845 & 0.062732 \\
\hline & H5'2 & 0.142819 & 0.120952 & 0.127732 & 0.152672 & & HD2 & 0.075806 & 0.072694 & 0.08996 & 0.070117 \\
\hline & H5'3 & 0.126262 & 0.132352 & 0.133563 & 0.120414 & & HD3 & 0.097543 & 0.10889 & 0.09718 & 0.112263 \\
\hline \multirow{2}{*}{\multicolumn{2}{|c|}{ Sum }} & 0.988574 & 0.998749 & 0.985757 & 0.983995 & \multicolumn{2}{|c|}{ Sum } & 0.676403 & 0.78195 & 0.823062 & 0.688375 \\
\hline & & & & & & \multicolumn{2}{|c|}{ Guanidine group } & 0.492679 & 0.598976 & 0.612487 & 0.510503 \\
\hline
\end{tabular}

a) See the structure Figure S2. 

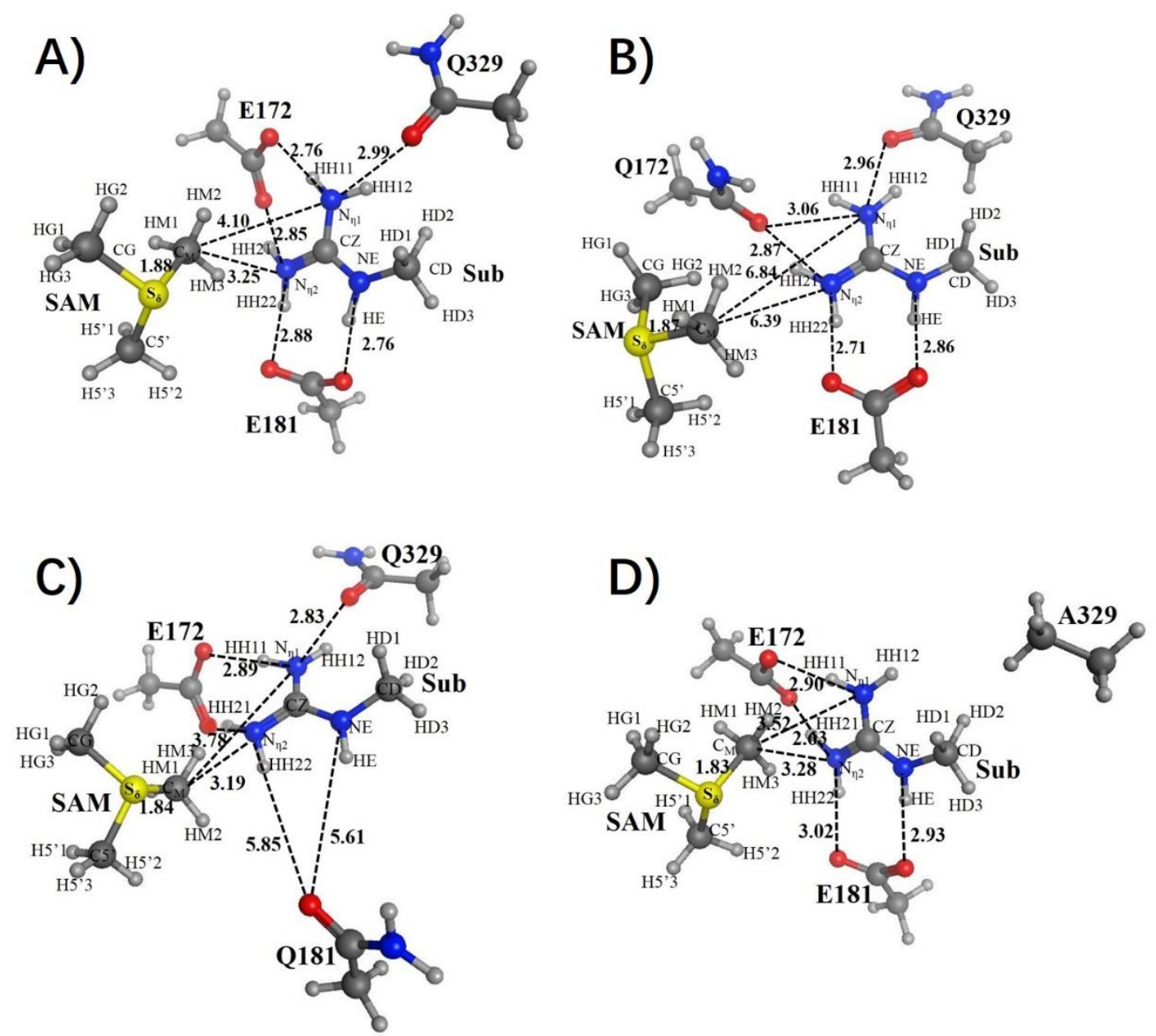

Figure S2. The cluster models of (A) wild-type PRMT7, (B) E172Q, (C) E181Q and (D) Q329A. For each cluster model, the representative active-site structures and key residues were based on the structures from QM/MM molecular dynamics simulations. 
The representative active-site structures for the reactant state and near transition state as well as the free energy profiles for the $2^{\text {nd }}$ methylations
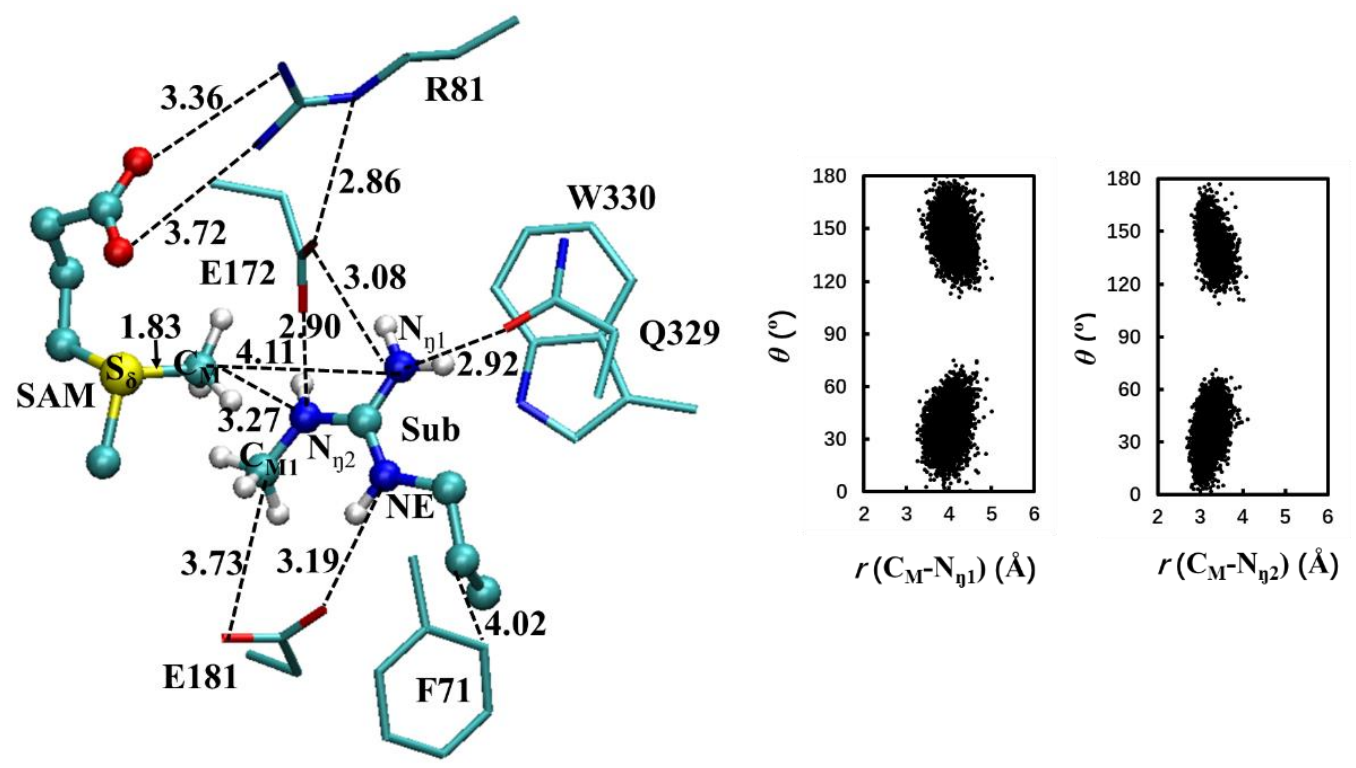

Figure S3. Representative active site structure of the reactant complex I for $2^{\text {nd }}$ methylation along with $r\left(\mathrm{C}_{\mathrm{M}} \cdots \mathrm{N}_{\mathfrak{n}}\right)$ and $\theta$ distributions obtained from the QM/MM simulation. (left) $2^{\text {nd }}$ methylation on $\mathrm{N}_{\mathfrak{y} 1}$. (right) $2^{\text {nd }}$ methylation on $\mathrm{N}_{\mathfrak{y} 2}$. 
A)

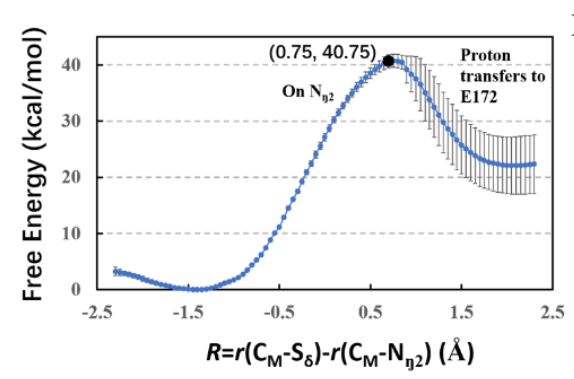

D)

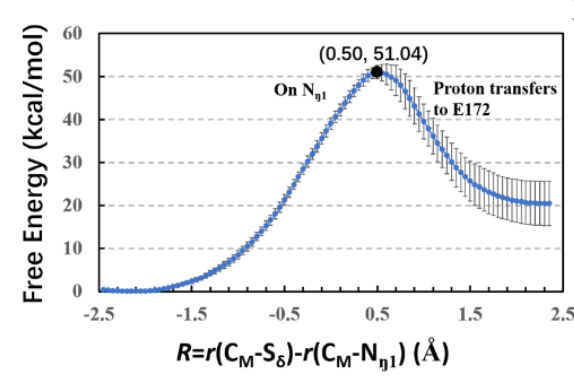

B)

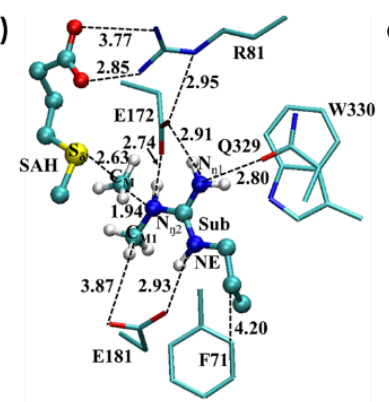

E)

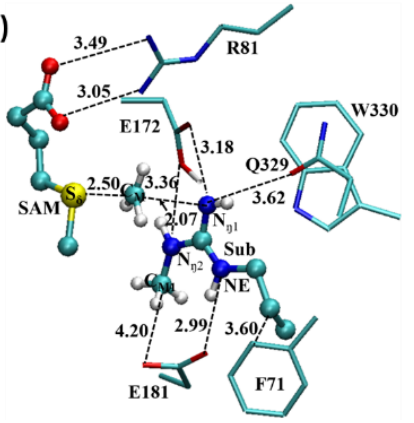

C)

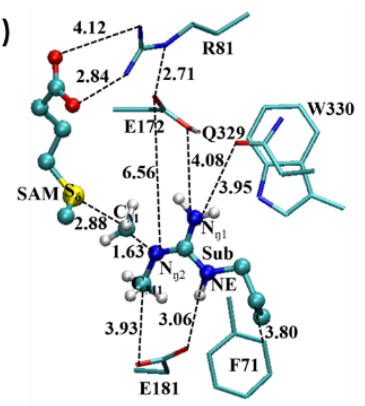

Figure S4. (A) Free energy profile for the $2^{\text {nd }}$ methylation to $\mathrm{N}_{\mathrm{y} 2}$ in complex I. (B) Representative active site structures of near TS for the $2^{\text {nd }}$ methylation to $\mathrm{N}_{\mathrm{n} 2}$ obtained from the free energy simulations. (C) Representative active site structures of (TS+1) state for the $2^{\text {nd }}$ methylation to $\mathrm{N}_{\mathrm{n} 2}$ as well as the proton transfer to E172. (D) Free energy profile for the $2^{\text {nd }}$ methylation to $\mathrm{N}_{\mathfrak{n} 1}$. (E) Representative active site structures of near TS for the $2^{\text {nd }}$ methylation to $\mathrm{N}_{\mathfrak{n} 1}$ as well as the proton transfer to E172. 

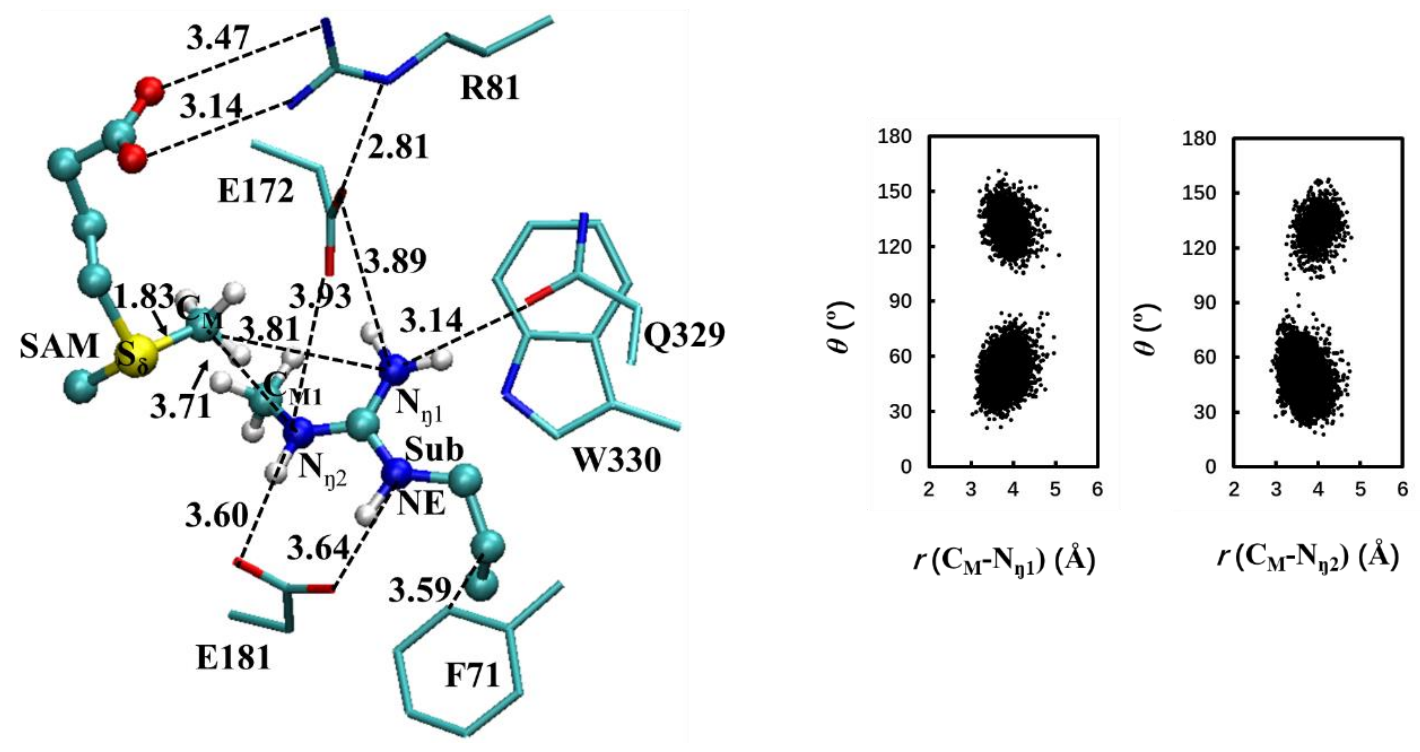

Figure S5. Representative active site structure of the reactant complex II for $2^{\text {nd }}$ methylation along with $r\left(\mathrm{C}_{\mathrm{M}} \cdots \mathrm{N}_{\mathfrak{y}}\right)$ and $\theta$ distributions obtained from the QM/MM simulation. (left) $2^{\text {nd }}$ methylation on $\mathrm{N}_{\mathrm{n} 1}$. (right) $2^{\text {nd }}$ methylation on $\mathrm{N}_{\mathrm{n} 2}$. 


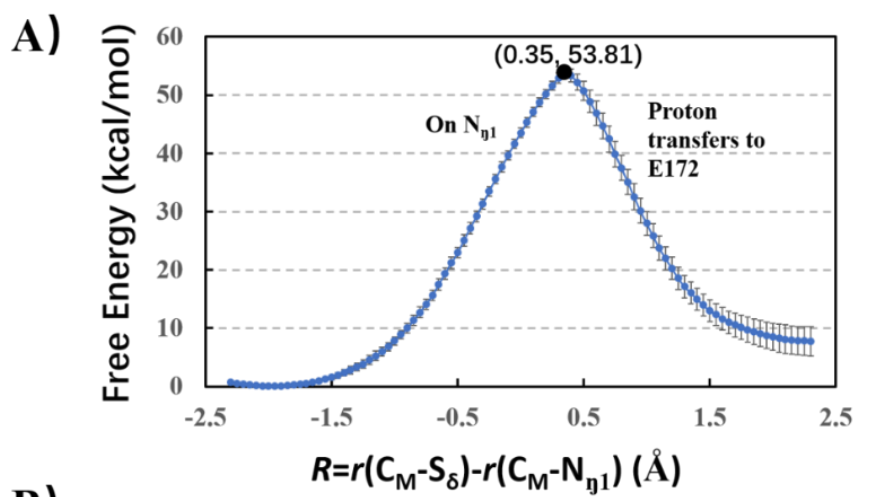

B)

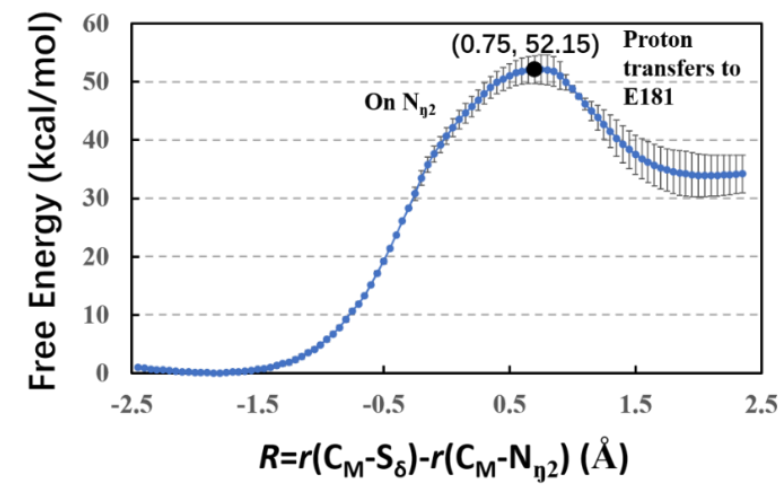

Figure S6. Free energy profile for the $2^{\text {nd }}$ methylation addition to (A) the $\mathrm{N}_{\mathrm{n} 1}$ atom and (B) the $\mathrm{N}_{\mathrm{y} 2}$ atom in complex II. 

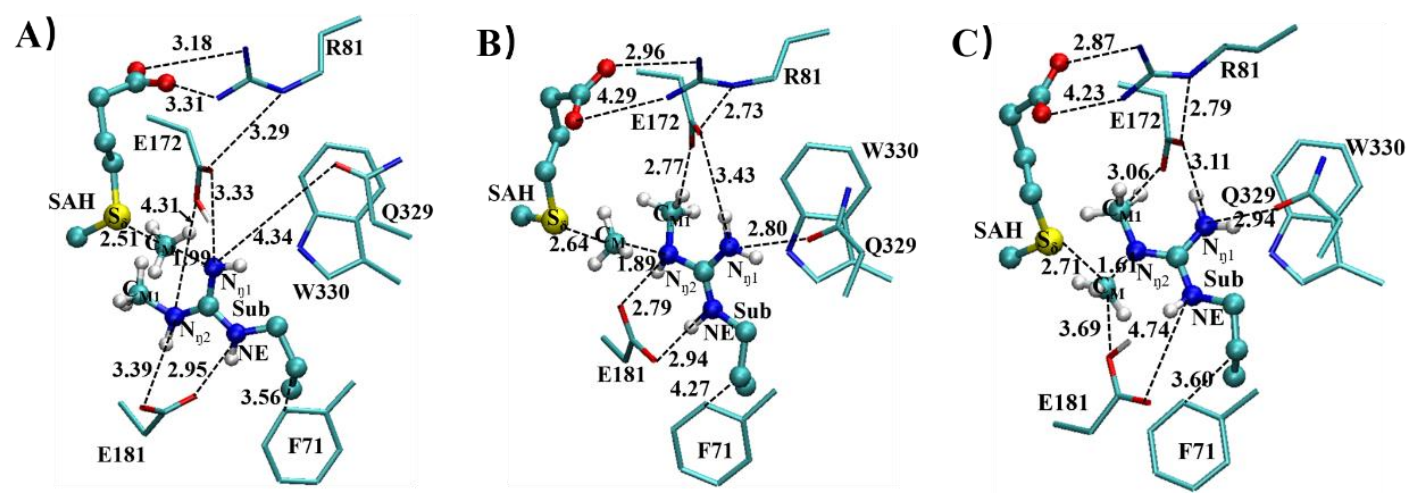

Figure S7. (A) Representative active site structures of near TS for the $2^{\text {nd }}$ methylation to $\mathrm{N}_{\mathrm{y} 1}$ in complex II obtained from the free energy simulations. (B) Representative active site structures of near TS for the $2^{\text {nd }}$ methylation to $\mathrm{N}_{\mathrm{n} 2}$ in complex II obtained from the free energy simulations. (C) Representative active site structures of $(\mathrm{TS}+2)$ state for the $2^{\text {nd }}$ methylation to $\mathrm{N}_{\mathrm{n} 2}$ in complex II obtained from the free energy simulations. 


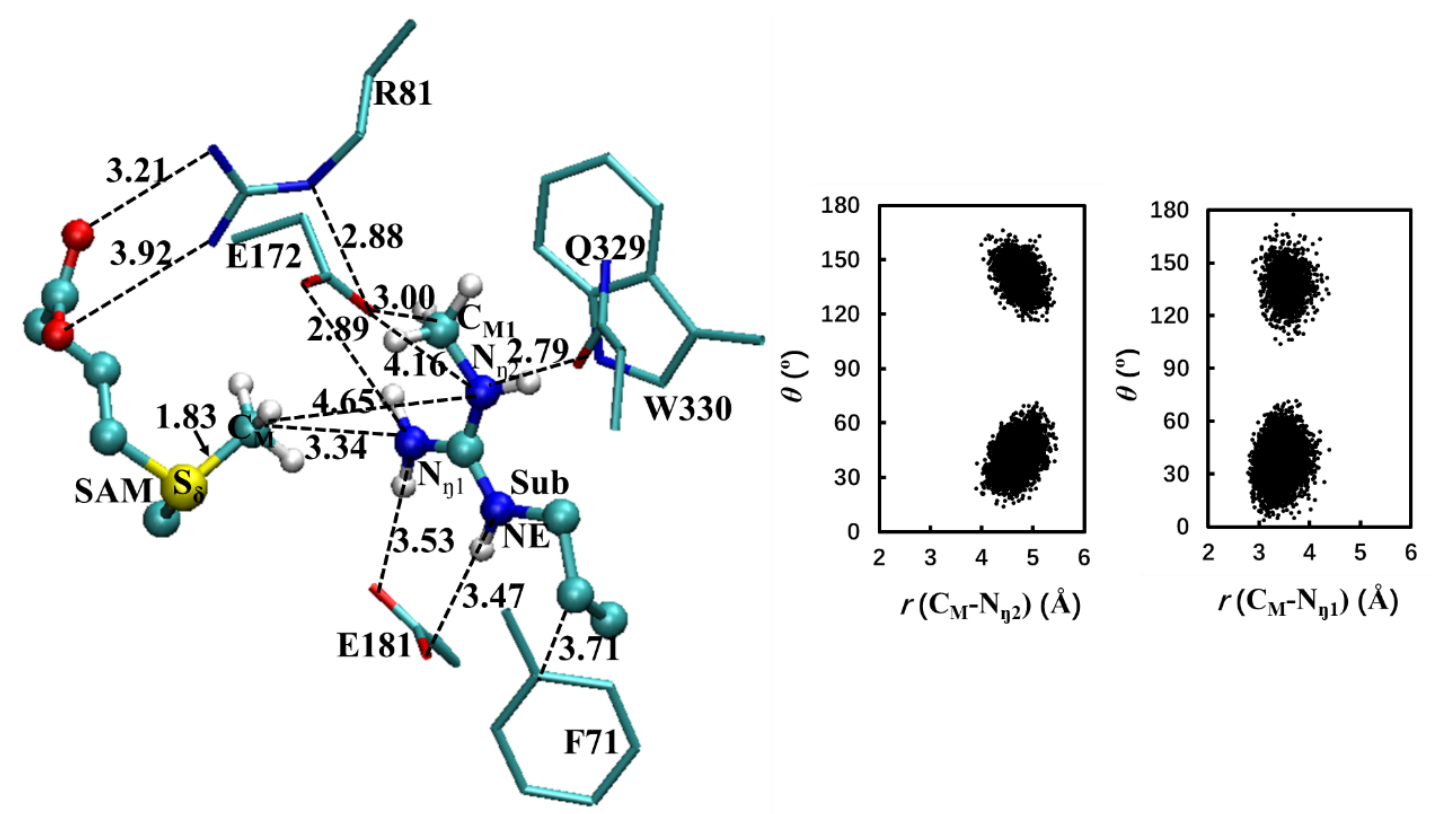

Figure S8. Representative active site structure of the reactant complex III for $2^{\text {nd }}$ methylation along with $r\left(\mathrm{C}_{\mathrm{M}} \cdots \mathrm{N}_{\mathrm{n}}\right)$ and $\theta$ distributions obtained from the QM/MM simulation. (left) $2^{\text {nd }}$ methylation on $\mathrm{N}_{\mathrm{n} 2}$. (right) $2^{\text {nd }}$ methylation on $\mathrm{N}_{\mathrm{n} 1}$. 


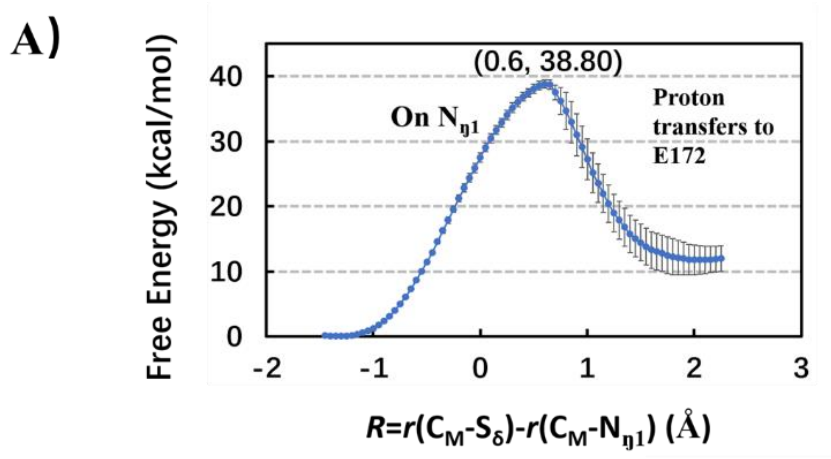

B)

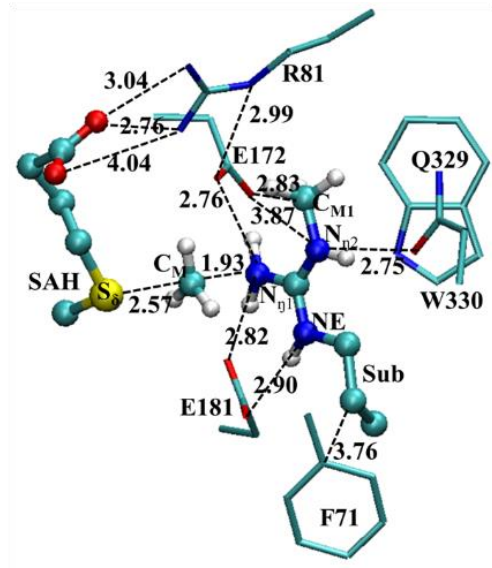

C)

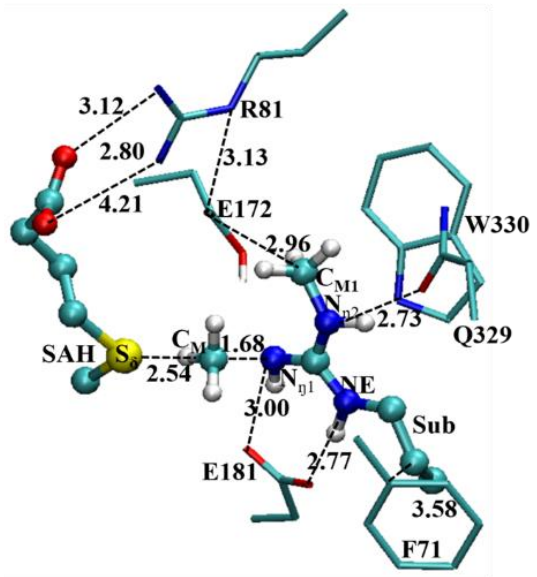

Figure S9. (A) Free energy profile for the $2^{\text {nd }}$ methylation to $\mathrm{N}_{\mathfrak{n} 1}$ in complex III. (B) Representative active site structures of near TS for the $2^{\text {nd }}$ methylation to $\mathrm{N}_{\mathrm{n} 1}$ in complex III obtained from the free energy simulations. (C) Representative active site structures of $(\mathrm{TS}+2)$ state for the $2^{\text {nd }}$ methylation to $\mathrm{N}_{\mathfrak{n} 1}$ in complex III obtained from the free energy simulations. 


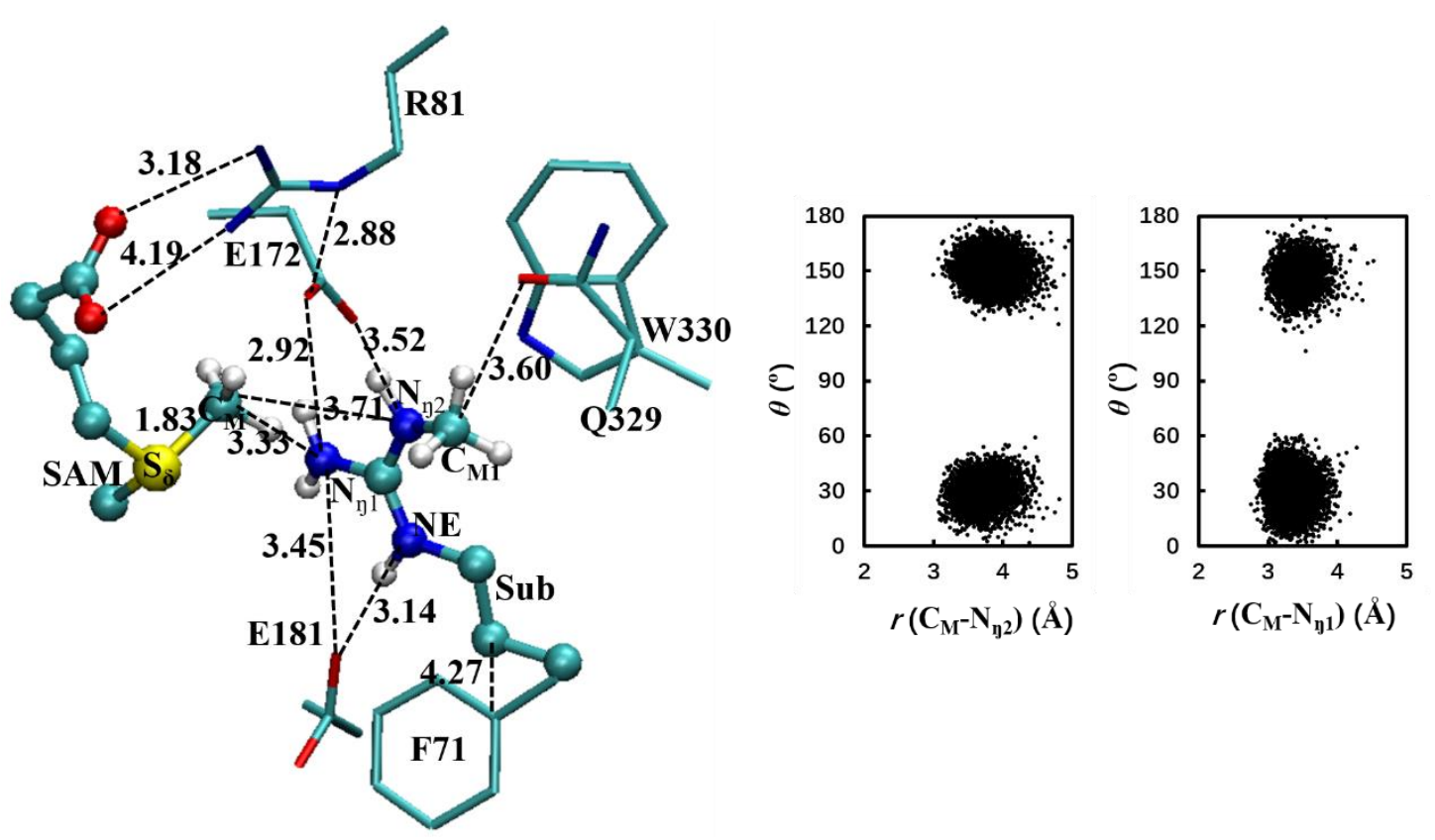

Figure S10. Representative active site structure of the reactant complex IV for $2^{\text {nd }}$ methylation along with $r\left(\mathrm{C}_{\mathrm{M}} \cdots \mathrm{N}_{\mathrm{y}}\right)$ and $\theta$ distributions obtained from the QM/MM simulation. (left) $2^{\text {nd }}$ methylation on $\mathrm{N}_{\mathrm{n} 2}$. (right) $2^{\text {nd }}$ methylation on $\mathrm{N}_{\mathrm{n} 1}$. 
A)

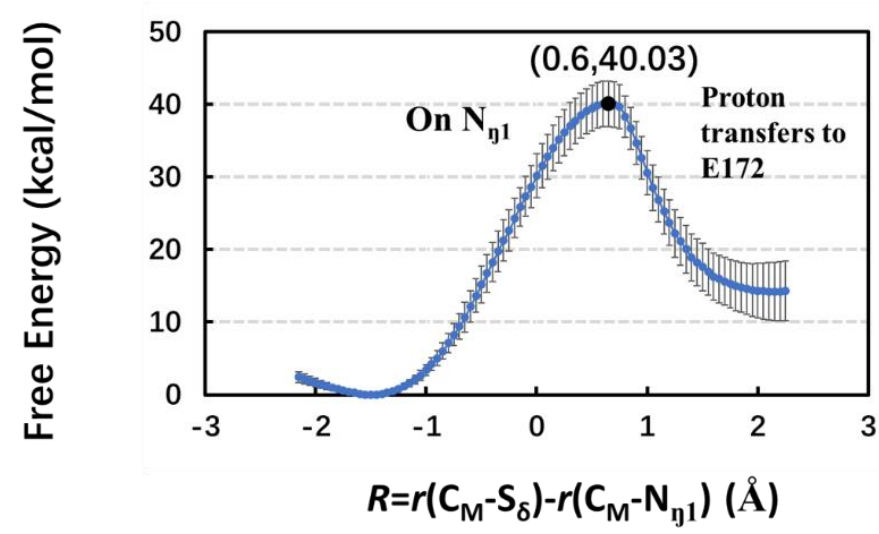

B)

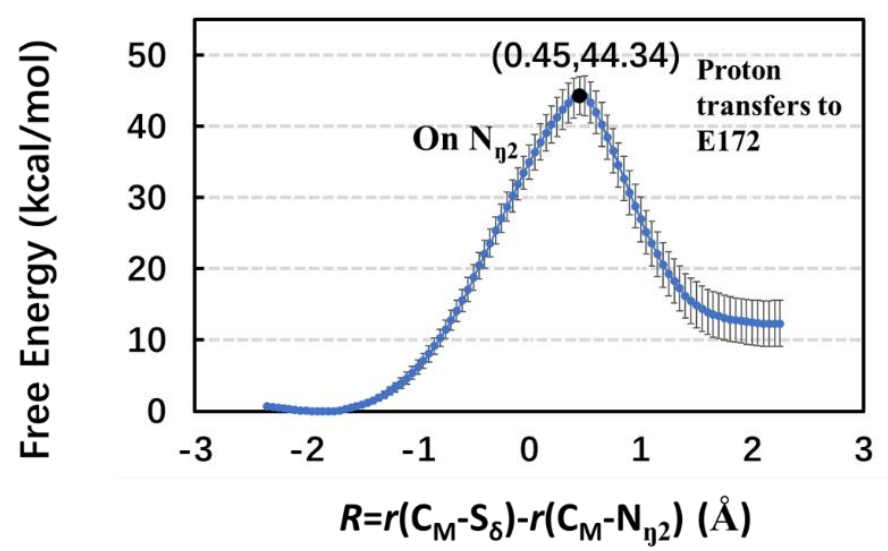

Figure S11. (A) Free energy profile for the $2^{\text {nd }}$ methylation to $\mathrm{N}_{\mathrm{y} 1}$ in complex IV. (B) Free energy profile for the $2^{\text {nd }}$ methylation to $\mathrm{N}_{\mathrm{n} 2}$ in complex IV. 
A)
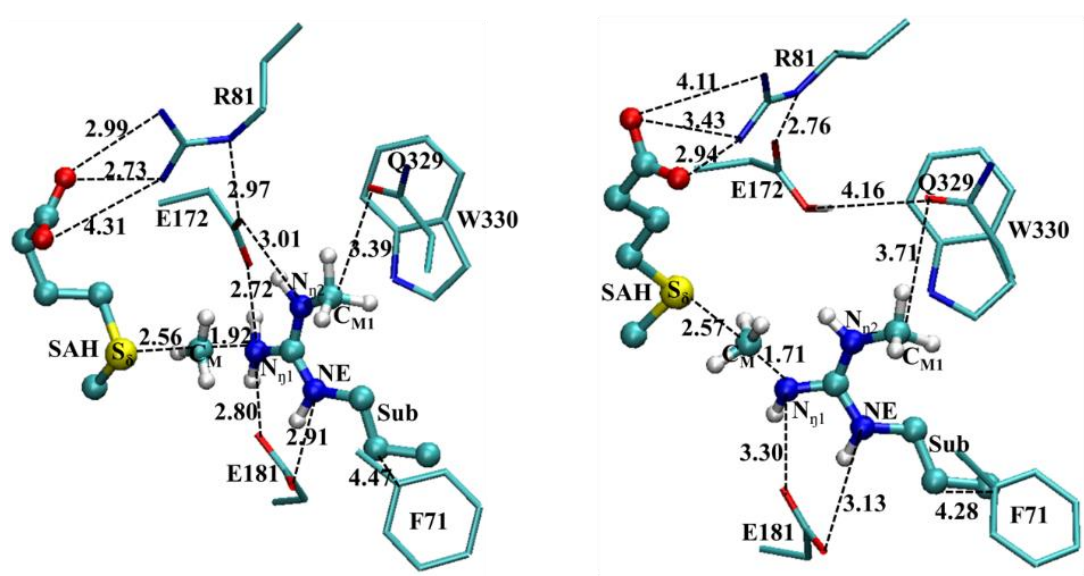

B)
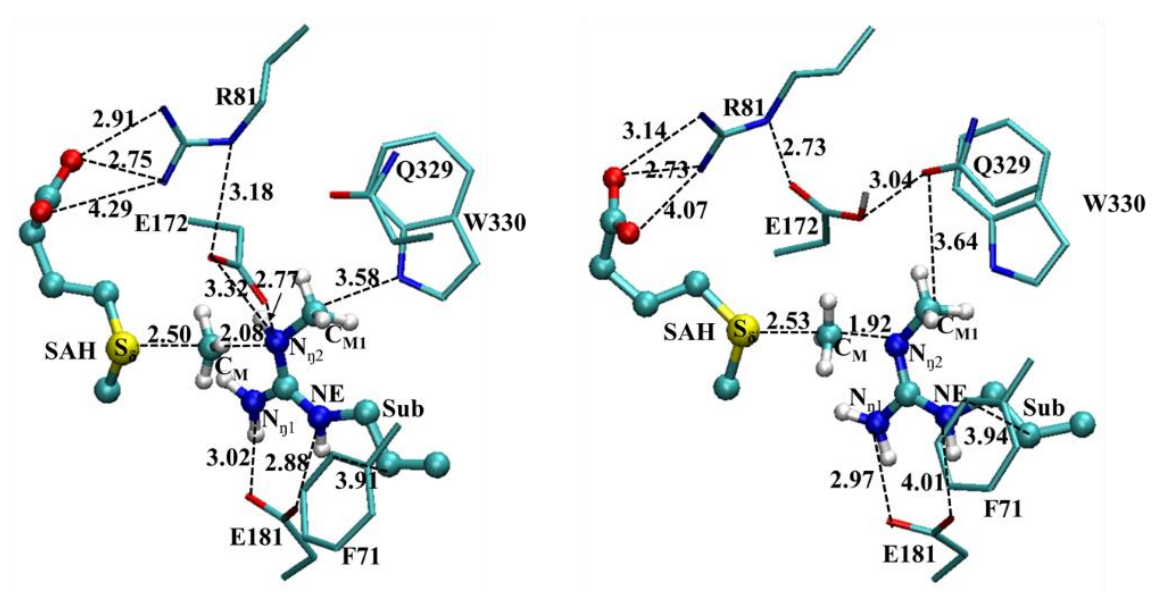

Figure S12. (A) Representative active site structures of near TS for the $2^{\text {nd }}$ methylation to $N_{\mathfrak{y} 1}$ in complex IV obtained from the free energy simulations. (B) Representative active site structures of (TS+1) state for the $2^{\text {nd }}$ methylation to $\mathrm{N}_{\mathrm{n} 1}$ in complex IV obtained from the free energy simulations. (C) Representative active site structures of near TS for the $2^{\text {nd }}$ methylation to $\mathrm{N}_{\mathrm{n} 2}$ in complex IV obtained from the free energy simulations. (D) Representative active site structures of $(\mathrm{TS}+2)$ state for the $2^{\text {nd }}$ methylation to $\mathrm{N}_{\mathrm{n} 2}$ in complex IV obtained from the free energy simulations. 